\title{
iPTF SEARCH FOR AN OPTICAL COUNTERPART TO GRAVITATIONAL-WAVE TRANSIENT GW150914
}

\author{
M. M. Kasliwal ${ }^{1}$, S. B. Cenko ${ }^{2,3}$, L. P. $\operatorname{Singer}^{2,20}$, A. Corsi ${ }^{4}$, Y. CaO ${ }^{1}$, T. Barlow ${ }^{1}$, V. Bhalerao ${ }^{5}$, E. Bella $^{1}$, D. Cook ${ }^{1}$, \\ G. E. Duggan ${ }^{1}$, R. Ferretti ${ }^{6}$, D. A. Frail $^{7}$, A. Horesh ${ }^{8}$, R. Kendrick ${ }^{9}$, S. R. Kulkarni ${ }^{1}$, R. Lunnan ${ }^{1}$, N. Palliyaguru ${ }^{4}$, \\ ${\text { R. } \text { Laher }^{10} \text {, F. Masci }}^{11}$, I. Manulis ${ }^{8}$, A. A. Miller ${ }^{1,12,21}$, P. E. Nugent ${ }^{13,14}$, D. Perley ${ }^{15}$, T. A. Prince ${ }^{1}$, R. M. Quimby ${ }^{16,17}$, \\ J. Rana ${ }^{5}$, U. Rebbapragada ${ }^{12}$, B. Sesar ${ }^{18}$, A. Singhal ${ }^{5}$, J. Surace ${ }^{10}$, And A. Van Sistine ${ }^{19}$ \\ ${ }^{1}$ Cahill Center for Astrophysics, California Institute of Technology, Pasadena, CA 91125, USA \\ ${ }^{2}$ Astrophysics Science Division, NASA Goddard Space Flight Center, Code 661, Greenbelt, MD 20771, USA \\ ${ }^{3}$ Joint Space-Science Institute, University of Maryland, College Park, MD 20742, USA \\ ${ }^{4}$ Texas Tech University, Physics Department, Lubbock, TX 79409-1051, USA \\ ${ }^{5}$ Inter-University Centre for Astronomy and Astrophysics (IUCAA), Post Bag 4, Ganeshkhind, Pune 411007, India \\ ${ }^{6}$ The Oskar Klein Centre, Department of Physics, Stockholm University, SE-106 91 Stockholm, Sweden \\ ${ }^{7}$ National Radio Astronomy Observatory, Socorro, NM, USA \\ ${ }^{8}$ Department of Particle Physics and Astrophysics, Weizmann Institute of Science, 76100 Rehovot, Israel \\ ${ }^{9}$ Lockheed Martin Space Systems Company, Palo Alto, CA, USA \\ ${ }^{10}$ Spitzer Science Center, California Institute of Technology, M/S 314-6, Pasadena, CA 91125, USA \\ ${ }^{11}$ Infrared Processing and Analysis Center, California Institute of Technology, Pasadena, CA 91125, USA \\ 12 Jet Propulsion Laboratory, California Institute of Technology, Pasadena, CA 91109, USA \\ ${ }^{13}$ Astronomy Department, University of California at Berkeley, Berkeley, CA 94720, USA \\ ${ }_{15}^{14}$ Lawrence Berkeley National Laboratory, 1 Cyclotron Road, MS 50B-4206, Berkeley, CA 94720, USA \\ ${ }^{15}$ Dark Cosmology Centre, Niels Bohr Institute, Juliane Maries Vej 30, Copenhagen, DK-2100, Denmark \\ ${ }^{16}$ San Diego State University, San Diego, CA, USA \\ ${ }^{17}$ Kavli IPMU (WPI), UTIAS, The University of Tokyo, Kashiwa, Chiba 277-8583, Japan \\ ${ }^{18}$ Max Planck Institute for Astronomy, Königstuhl 17, D-69117 Heidelberg, Germany \\ ${ }^{19}$ Department of Physics, University of Wisconsin-Milwaukee, Milwaukee, WI 53201, USA \\ Received 2016 February 28; revised 2016 May 25; accepted 2016 May 26; published 2016 June 17
}

\begin{abstract}
The intermediate Palomar Transient Factory (iPTF) autonomously responded to and promptly tiled the error region of the first gravitational-wave event GW150914 to search for an optical counterpart. Only a small fraction of the total localized region was immediately visible in the northern night sky, due both to Sun-angle and elevation constraints. Here, we report on the transient candidates identified and rapid follow-up undertaken to determine the nature of each candidate. Even in the small area imaged of $126 \mathrm{deg}^{2}$, after extensive filtering, eight candidates were deemed worthy of additional follow-up. Within two hours, all eight were spectroscopically classified by the Keck II telescope. Curiously, even though such events are rare, one of our candidates was a superluminous supernova. We obtained radio data with the Jansky Very Large Array and X-ray follow-up with the Swift satellite for this transient. None of our candidates appear to be associated with the gravitational-wave trigger, which is unsurprising given that GW150914 came from the merger of two stellar-mass black holes. This end-to-end discovery and follow-up campaign bodes well for future searches in this post-detection era of gravitational waves.
\end{abstract}

Key words: gravitational waves - methods: observational - techniques: spectroscopic - surveys

\section{INTRODUCTION}

The direct detection of gravitational waves (GWs) marks the dawn of a new era (Abbott et al. 2016b). It is widely agreed that the detection and study of the anticipated electromagnetic (EM) counterparts will vastly enrich the science returns for the field of GW astronomy. The photometric discovery of the EM counterpart will give a precise location and a spectrum of the host galaxy will give a precise redshift. This will enable a more accurate measurement of basic astrophysical properties such as the luminosity and energetics of this strong-field gravity event. If the spectrum is timely, it may also solve the long-standing mystery of the unknown sites of r-process nucleosynthesis.

The inherent challenge is that the two advanced GW interferometers, due to the low frequency of operation, give very poor on-sky localization (Abbott et al. 2013; Kasliwal \& Nissanke 2014; Singer et al. 2014; Berry et al. 2015). Nevertheless, the prospect of finding EM counterparts by searching large sky areas is promising as the search methodology is steadily

\footnotetext{
${ }^{20}$ NASA Postdoctoral Program Fellow.

${ }^{21}$ Hubble Fellow.
}

improving-from early efforts in the enhanced LIGO S6 run (Aasi et al. 2014), to proof-of-concept localizations of coarse Fermi gamma-ray bursts (Singer et al. 2013, 2015), to a score of EM facilities promptly responding to GW150914 (Abbott et al. 2016a).

At the time of the GW150914 trigger, there was no information disclosed on the nature of the event, i.e., whether it was a binary black hole merger or binary neutron star merger or something else (GCN 18330). Many facilities undertook a search for an EM counterpart (e.g., Connaughton et al. 2016; Evans et al. 2016; Smartt et al. 2016; Soares-Santos et al. 2016). Months later, after offline analysis, the event was identified as a binary black hole merger (GCN 18858).

Here, we present the intermediate Palomar Transient Factory (iPTF) follow-up effort. iPTF uses the Samuel Oschin 48 inch telescope on Palomar mountain equipped with the CFH12K camera with a field of view of $7.1 \mathrm{deg}^{2}$ (Law et al. 2009). Our motivation was to look for an optical counterpart powered by free neutron decay (Metzger et al. 2015), or heavy element radioactive decay (Metzger \& Fernández 2014; Kasen et al. 2015). We describe the sky area coverage, candidate 


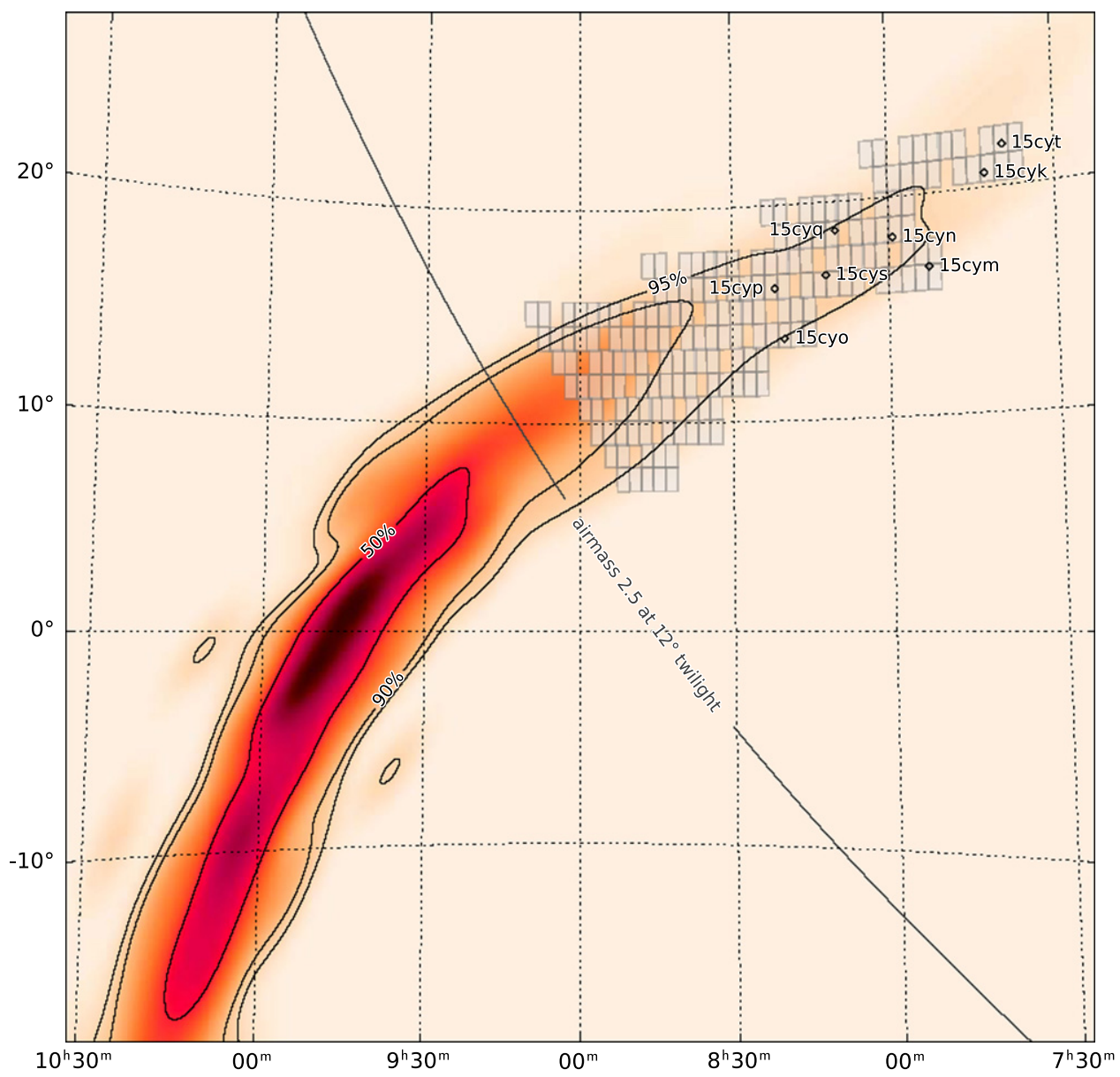

Figure 1. iPTF coverage map (gray tiles) of GW150914. The color coding and contours denote GW probability based on the initial map. Due to the Sun-angle and elevation constraints, we were only able to image the westernmost region of the localization. The enclosed probability was $2.5 \%$ in the initial map and $0.2 \%$ in the final map. Eight candidates were identified and classified.

identification, spectroscopic classification, and panchromatic follow-up. We conclude with our plans for a way forward.

\section{IDENTIFYING CANDIDATES}

On UT 2015 September 16 03:17, the iPTF Target of Opportunity Marshal automatically responded to the gravitational-wave trigger alert G184098 (later named GW150914). It immediately notified the team via phone calls and SMS alerts that there had been a $\mathrm{GW}$ trigger. It also computed that due to the Sun-angle constraint and elevation constraints, iPTF would only be able to access $2.5 \%$ of the enclosed probability in the initial map by tiling $126 \mathrm{deg}^{2}$ just before sunrise at high airmass (Figure 1). This total area calculation takes into account the two non-working CCDs and the gaps between the CCDs. The small containment probability was because the southern mode of the updated ("LIB") localization was too far south to be observable from Palomar, whereas most of the northern mode rose only after $12^{\circ}$ morning twilight. Clouds did not cooperate and the Palomar 48 inch dome remained closed the first night after trigger. However, the next night (UT September 17), we imaged 18 fields covering this area with exposures of $1 \mathrm{~min}$ using the R-band filter (see details in Table 3; GCN 18337). The scheduling and choice of tiles was further optimized applying the algorithm described in Rana et al. (2016). A second epoch with a baseline separation of 30 minutes ( \pm 1 minutes) was obtained for 13 fields.

Within minutes of obtaining the data, our automated real-time image subtraction pipeline started loading candidates into our database. We have two independent, real-time pipelines-one running at the National Energy Research Scientific Computing Center (NERSC) using the HOTPANTS image subtraction algorithm (Nugent et al. 2015) and the other running at the Infrared Processing and Analysis Center (IPAC) using the PTFIDE algorithm (F. Masci et al. 2016, in preparation). Due to the dynamic nature of the optical sky, the candidate list was dominated by false-positive transients unrelated to the gravitational-wave trigger. A total of 127,676 candidates were loaded into the NERSC database and 32,576 in the IPAC database. Our automated machine-learning-aided filtering algorithms rejected the moving objects in our solar system, variable stars in the Milky Way as well as subtraction artifacts. A list of 13 candidates was presented on a dynamic web portal for human vetting.

We have been refining our software algorithms that quickly sift through the large number of candidates during our Fermi Gamma-ray Burst Monitor afterglow search effort (Singer et al. 2015). The EM-GW challenge has some similarities and some differences. The similarities are that we need to continue to reject foreground asteroids/variable stars and background supernovae/active galactic nuclei. The differences are that compared to a gamma-ray burst afterglow, the EM-GW 
Table 1

Candidates Flagged for Follow-up

\begin{tabular}{|c|c|c|c|c|c|c|c|}
\hline Name & RA (J2000) & DEC (J000) & Discovery Time & Mag (R-band) & Minutes to Spectrum & Classification & Redshift \\
\hline iPTF15суо & $8^{\mathrm{h}} 19^{\mathrm{m}} 56^{\mathrm{s}} .18$ & $+13 \mathrm{~d} 52^{\prime} 42^{\prime \prime} 0$ & 2015 Sep 17 05:54:55.6 & $17.75 \pm 0.01$ & 71 & SN Ia (SN1996X-like, +23d) & 0.029 \\
\hline iPTF15сур & $8^{\mathrm{h}} 21^{\mathrm{m}} 43^{\mathrm{s}} \cdot 68$ & $+16 \mathrm{~d} 12^{\prime} 42^{\prime \prime} 0$ & 2015 Sep 17 05:56:31.6 & $19.48 \pm 0.05$ & 125 & Nuclear & 0.028 \\
\hline iPTF15cys & $8^{\mathrm{h}} 11^{\mathrm{m}} 55^{\mathrm{s}} \cdot 59$ & $+16 \mathrm{~d} 43^{\prime} 10^{\prime \prime} 1$ & 2015 Sep 17 06:05:16.6 & $17.84 \pm 0.03$ & 46 & SN Ia (SN2004eo-like, $+22 \mathrm{~d}$ ) & 0.05 \\
\hline iPTF15cym & $7^{\mathrm{h}} 52^{\mathrm{m}} 35^{\mathrm{s}} \cdot 67$ & $+16 \mathrm{~d} 45^{\prime} 59^{\prime \prime} 6$ & 2015 Sep 17 05:46:17.1 & $19.88 \pm 0.20$ & 113 & SN II (SN1999M-like, +5d) & 0.055 \\
\hline iPTF15cyq & $8^{\mathrm{h}} 10^{\mathrm{m}} 00^{\mathrm{s}} \cdot 86$ & $+18 \mathrm{~d} 42^{\prime} 18^{\prime \prime} 1$ & 2015 Sep 17 05:57:16.3 & $20.05 \pm 0.10$ & 39 & SN II (SN2004et-like, +47d) & 0.063 \\
\hline iPTF15cyn & $7^{\mathrm{h}} 59^{\mathrm{m}} 14^{\mathrm{s}} .93$ & $+18 \mathrm{~d} 12^{\prime} 54^{\prime \prime} 9$ & 2015 Sep 17 05:47:20.5 & $20.34 \pm 0.28$ & 124 & Nuclear & 0.062 \\
\hline iPTF15cyt & $7^{\mathrm{h}} 38^{\mathrm{m}} 59^{\mathrm{s}} \cdot 35$ & $+21 \mathrm{~d} 45^{\prime} 43 !^{\prime \prime} 2$ & 2015 Sep 17 06:08:09.3 & $19.65 \pm 0.09$ & 82 & Nuclear & 0.078 \\
\hline iPTF15cyk & $7^{\mathrm{h}} 42^{\mathrm{m}} 14^{\mathrm{s}} .87$ & $+20 \mathrm{~d} 36^{\prime} 43 !^{\prime \prime} 4$ & 2015 Sep 17 05:38:38.3 & $20.28 \pm 0.12$ & 97 & SLSN I (LSQ12dlf-like, +16d) & 0.539 \\
\hline
\end{tabular}



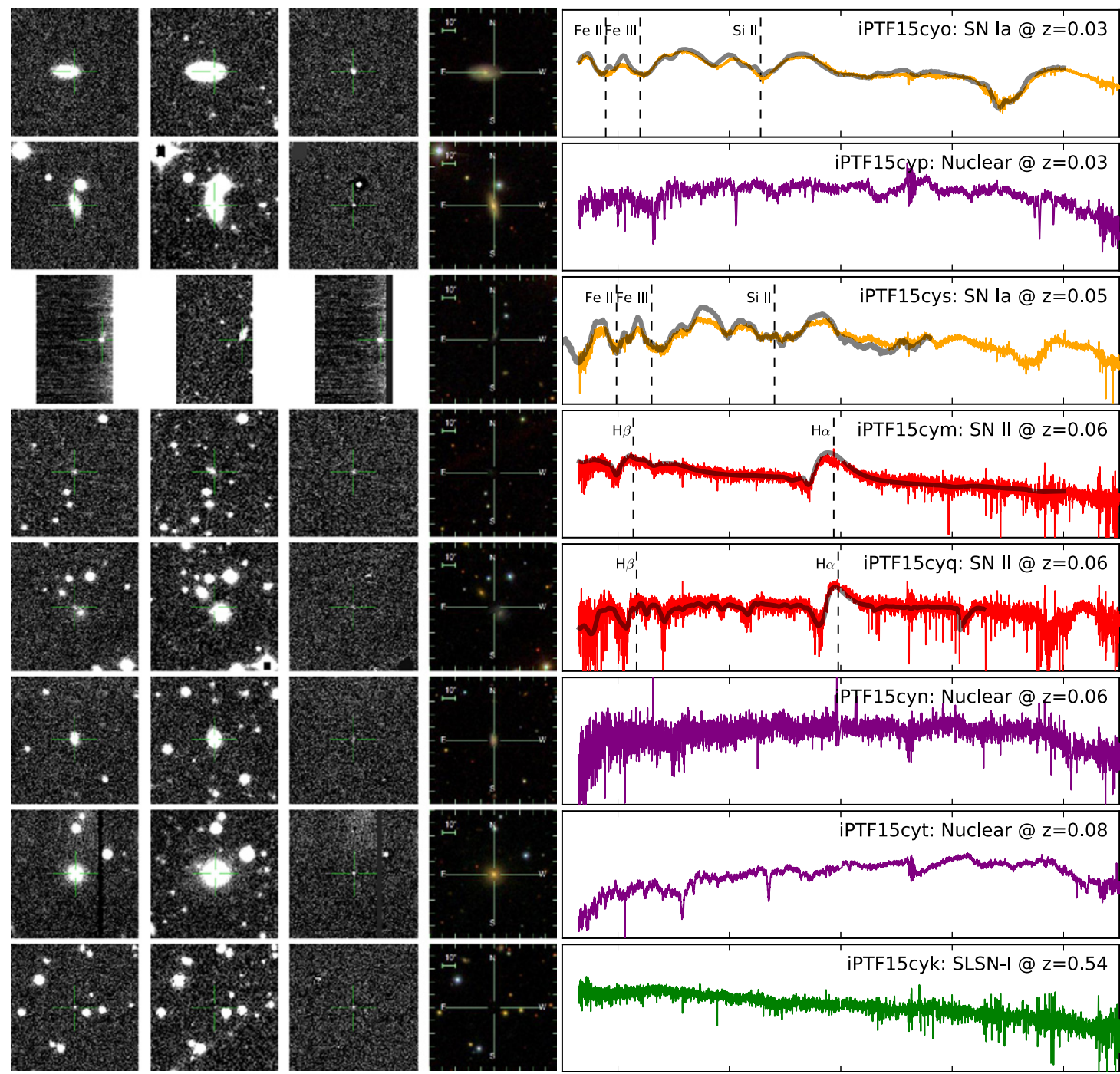

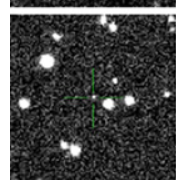

New
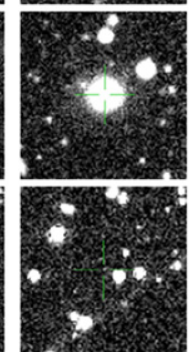

Ref

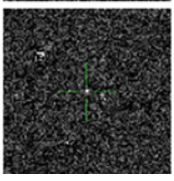

Sub

\section{SDSS}

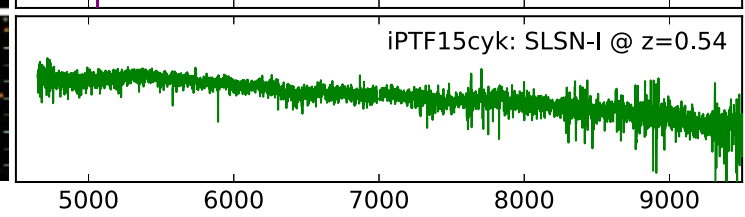

Figure 2. Keck II/DEIMOS classification spectra of eight iPTF candidates obtained within two hours of discovery. Also shown, from left to right, the P48 discovery image, reference image, subtraction image, and SDSS thumbnail around each candidate location. Colors denote spectroscopic class: SN Ia (red), SN II (blue), Nuclear (purple), SLSN I (green). Overplotted in gray lines is the best match from a supernova spectra library (SN1996X for iPTF15cyo, SN2004eo for iPTF15cys, SN1999M for iPTF15cym, SN2004et for IPTF15cyq). Additional follow-up data were needed to classify iPTF 15cyk as an SLSN I (see Figure 4).

counterpart may be relatively fainter and/or slower and/or redder. Knowing that the EM counterpart is relatively nearby due to the advanced LIGO sensitivity helps further reduce false positives.

The following are some rejection criteria:

1. Movement in detections in two epochs separated by at least 15 minutes suggesting the candidate is an asteroid.

2. Past history of eruption in PTF/iPTF data (baseline of six years) suggesting the candidate is an old transient.

3. Previously known radio source or X-ray source suggesting the candidate is an active galactic nucleus.

4. Previously known optical or infrared point source underneath the position suggesting the candidate is a stellar flare.

The following criteria lead to flags for follow-up spectroscopy, additional photometry, and/or multi-band follow-up:
1. Host galaxy (within $100 \mathrm{kpc}$ of transient) with spectroscopic redshift $<0.05$ (or photometric redshift $<0.1$ ) this is motivated by advanced LIGO's sensitivity limit to binary neutron star mergers.

2. Photometric evolution on hour timescale $(>0.2 \mathrm{mag})$ or day timescale $(>0.5 \mathrm{mag})$ or one-week timescale (>1 mag) - this serves as a strong discriminant against old supernovae. We note that this flag was not applied for GW150914 as all candidates of interest were spectroscopically classified within two hours.

3. Hostless candidates with no counterpart in deep iPTF reference co-adds - even though these are unlikely to be local, we flag these events as they are relatively rare.

To quantify the relative efficacy of each criterion, we discuss the most severe cuts in order of severity by applying each criterion independently. Of the 127,676 candidates in our 


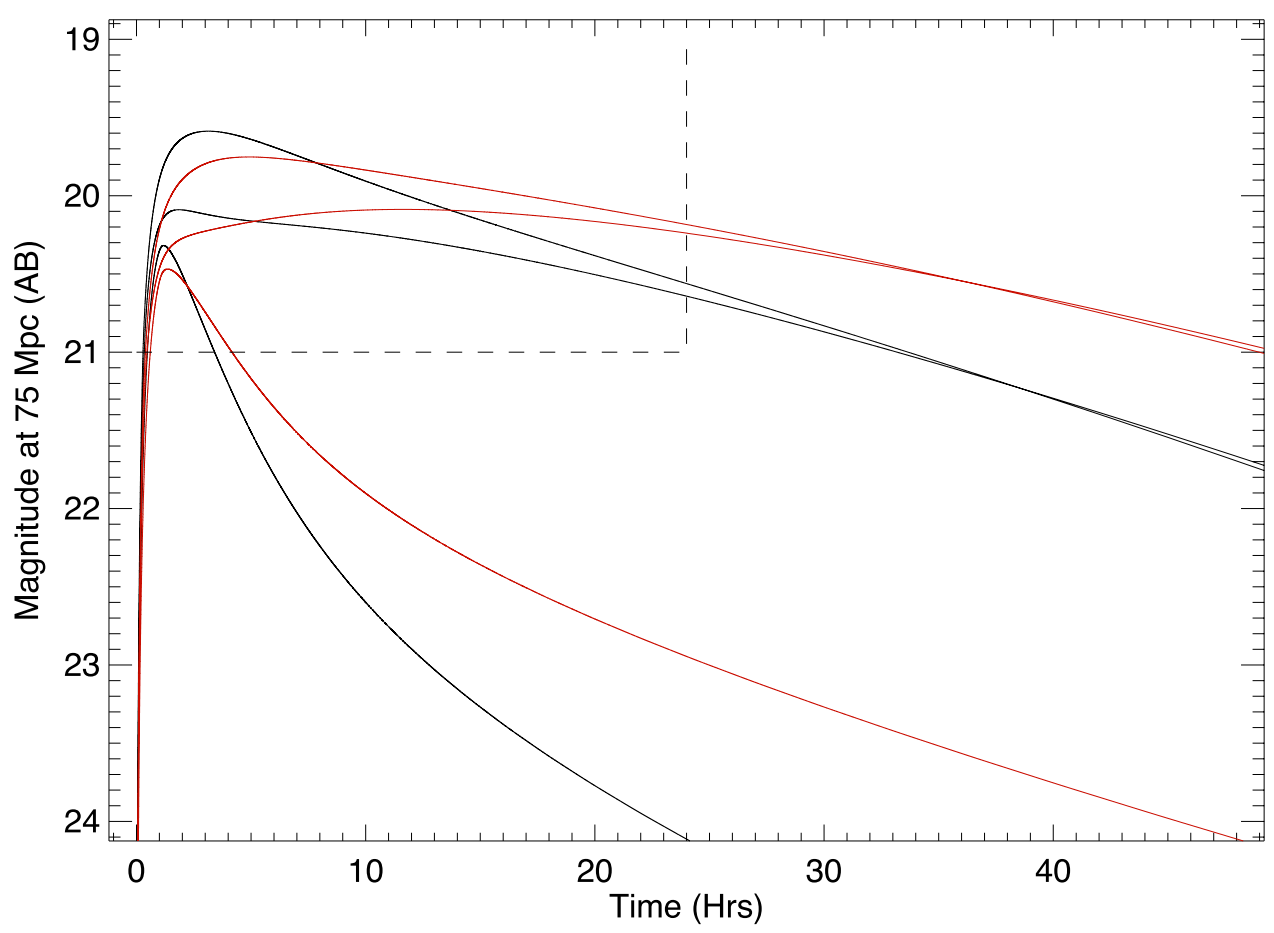

Figure 3. Predicted optical counterpart based on free neutron decay (Metzger et al. 2015). Black lines are g-band and red lines are r-band light curves at 75 Mpc (sensitivity limit of advanced LIGO to binary neutron star mergers in O1). The three curves assume three different values for opacity and neutron mass to represent the fast, intermediate, and slow light curve evolution cases, i.e., $\left(\kappa_{r}=30 \mathrm{~cm}^{2} \mathrm{gm}^{-1}, M_{n}=3 \times 10^{-5} \dot{M}_{\odot}\right),\left(\kappa_{r}=3 \mathrm{~cm}^{2} \mathrm{gm}^{-1}, M_{n}=3 \times 10^{-5} \dot{M}_{\odot}\right),\left(\kappa_{r}=3 \mathrm{~cm}^{2} \mathrm{gm}^{-1}\right.$, $\left.M_{n}=3 \times 10^{-4} \dot{M}_{\odot}\right)$. Note that the g-band is more luminous at peak but decays faster. Horizontal dashed line denotes the sensitivity of iPTF in $60 \mathrm{~s}$. Vertical dashed line denotes the timescale within which follow-up is undertaken by the GROWTH program.

Table 2

Panchromatic follow-up of Superluminous Supernova iPTF15cyk

\begin{tabular}{lccc}
\hline \hline Facility & $\begin{array}{c}\text { Epoch } \\
\text { UTC }\end{array}$ & Frequency & $\begin{array}{c}\text { Flux Limit } \\
\mathrm{erg} \mathrm{cm}^{-2} \mathrm{~s}^{-1} \mathrm{~Hz}^{-1}\end{array}$ \\
\hline Swift/XRT & 2015 Sep 18 18:12 & $2 \mathrm{keV}$ & $<4.5 \times 10^{-32}$ \\
VLA & $\begin{array}{c}\text { 2015 Oct 15 11:20:32- } \\
\text { 12:05:27 }\end{array}$ & $5.43 \mathrm{GHz}$ & $<2.6 \times 10^{-28}$ \\
VLA & 2015 Dec 06 04:52:11- & $5.43 \mathrm{GHz}$ & $<2.3 \times 10^{-28}$ \\
& $05: 00: 07$ & & \\
VLA & 2016 Jan 20 01:55:31- & $5.43 \mathrm{GHz}$ & $<2.3 \times 10^{-28}$ \\
& $02: 59: 40$ & & \\
\hline
\end{tabular}

NERSC pipeline, only 1007 candidates $(0.8 \%$ selection) are selected as being coincident with a galaxy within $200 \mathrm{Mpc}$, hence this is the most severe cut. 5803 candidates $(4.5 \%)$ are selected as passing our machine-learning cuts (we now have three generations of machine-learning algorithms; see details in Brink et al. 2013; Rebbapragada et al. 2014). 15,624 candidates $(12.2 \%)$ are selected as having two detections separated by 30 minutes in the same night. 78,951 candidates (62\% selection) are selected as not having an optical point source in the reference image. Similarly, in our IPAC pipeline, we had a total of 32,576 candidates. Of these, 24,699 did not match a star $(75.8 \%$ selection), 5302 had two detections (16.2\% selection), and 1964 passed our machine-learning cut (6.0\% selection).

In practice, these criteria are not all applied simultaneously and the candidates selected for human vetting are the result of a more complex database query. For example, prior to human vetting, we do not require coincidence with a nearby galaxy and we do not require any light curve properties. For the five fields where a second epoch was not completed on the same night, we did a manual search requiring a local universe match, and found two candidates that were both rejected as known asteroids. After human vetting of 13 candidates, 5 candidates were rejected as they showed past history of variability in the PTF data. In summary, our team flagged eight candidates for further follow-up in our marshal database (see Table 1). Next, we describe the prompt follow-up that was undertaken to investigate whether any of the candidates was associated with GW150914 (GCN 18341).

\section{SPECTROSCOPIC FOLLOW-UP}

Since Hawaii is west of Palomar Observatory, sunrise was three hours later and we were able to obtain spectra of all eight candidates in less than two hours from discovery (Figure 2). We emphasize that $\mathrm{PTF}$ has routinely been obtaining spectroscopic classification on the same night as discovery, totaling 165 transients with spectra within $12 \mathrm{hr}$, thus far. We observed with the DEep Imaging Multi-Object Spectrograph (DEIMOS; Faber et al. 2003) mounted on the Keck II telescope. We used the lowresolution $600 \mathrm{ZD}$ grating, giving spectral coverage between 4650 and $9600 \AA$ with a resolution of $3.5 \AA$ (FWHM). Our spectra are shown in Figure 2. A priori, since we searched $126 \mathrm{deg}^{2}$ to a depth of $20.5 \mathrm{mag}$, we expect $\approx 3.2$ supernovae using the rates in $\mathrm{Li}$ et al. (2011; and assuming that supernovae are brighter than -17 mag for 1 month, i.e., a volume out to $z=0.075$ ).

We cross-matched our spectra with a library of supernovae spectra augmenting the superfit software (Howell et al. 2005). Our classifications are in Table 1 . We found two Type Ia supernovae (SNe Ia), two hydrogen-rich core-collapse supernovae ( $\mathrm{SNe}$ II), three nuclear candidates (e.g., weak AGNs where the spectrum is dominated by the host galaxy), and one hostless transient with initially unclear classification (iPTF15cyk). Offline processing of the three nuclear candidates 


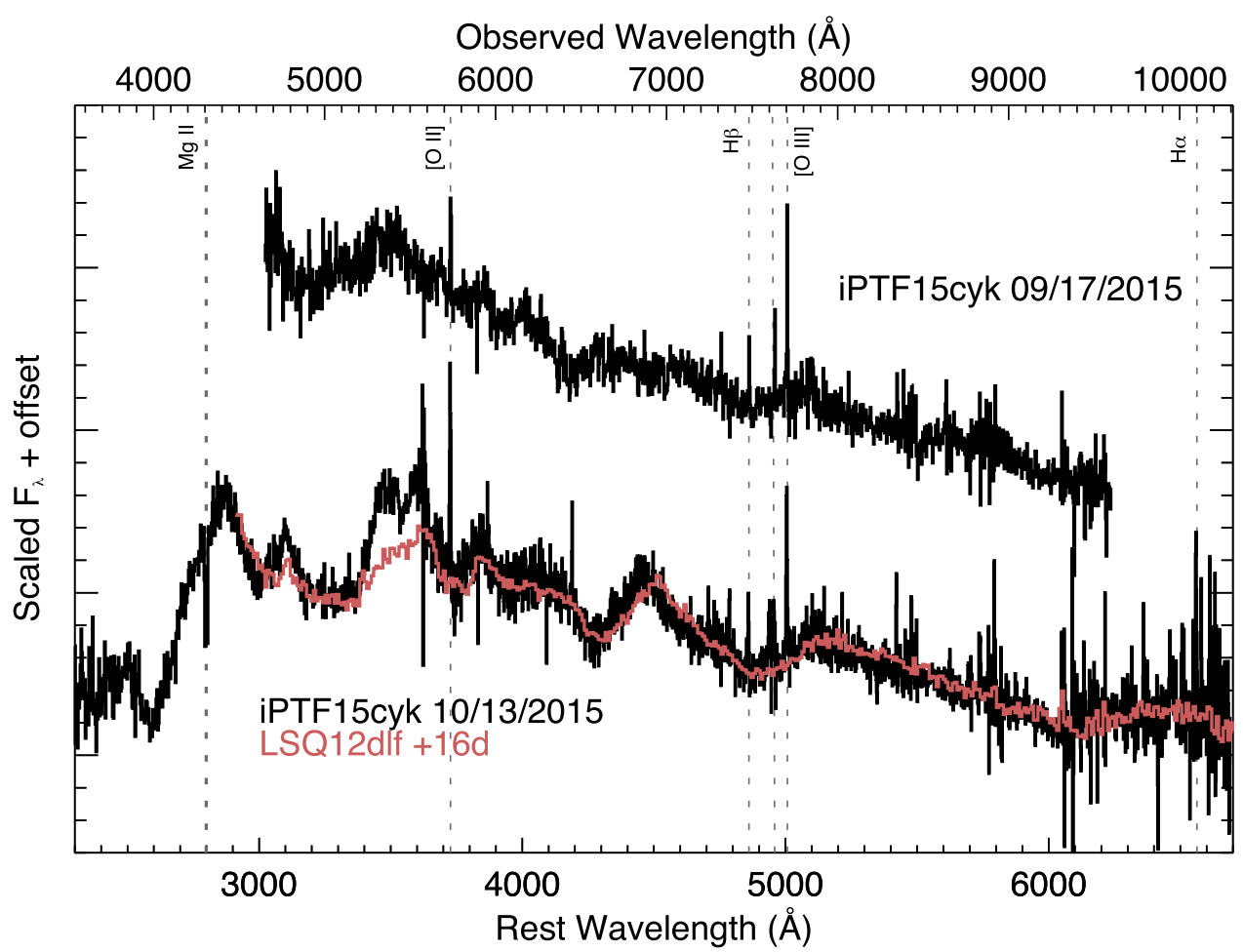

Figure 4. Spectral evolution of iPTF15cyk. The spectra show narrow lines from the host galaxy corresponding to $z=0.539$. The second spectrum matches a hydrogen-poor superluminous supernova, LSQ12dlf at $+16 \mathrm{~d}$.

also shows past history of photometric variability in the PTF data, which is consistent with the AGN hypothesis. The spectrum of iPTF15cyk was dominated by a blue continuum, with narrow lines suggesting a redshift of 0.539 (which would imply a very luminous transient). Since the nature of the $\mathrm{GW}$ source was unclear, we decided to obtain additional spectroscopic and multi-wavelength follow-up.

\section{RADIO AND X-RAY FOLLOW-UP}

We observed iPTF15cyk and the necessary calibrators with the Karl G. Jansky Very Large Array (VLA; Perley et al. 2009) in its $\mathrm{D}$ and DnC configurations. The observations were performed in the $\mathrm{C}$-band $(\approx 6 \mathrm{GHz}$ central frequency) under our Target of Opportunity program (VLA/15A-339; PI: Corsi). VLA data were reduced and imaged using the Common Astronomy Software Applications (CASA) package. In Table 2, we report the $3 \sigma$ upper limits derived for iPTF15cyk using the full $2 \mathrm{GHz}$ bandwidth (GCN 18914).

Given the host galaxy redshift, iPTF15cyk could be a superluminous supernova (SLSN) since the absolute magnitude at discovery was $-22 \mathrm{mag}$. Radio and X-ray emission from superluminous SNe may arise from interaction with the circumstellar medium (CSM; see, e.g., Ofek et al. 2013). In an alternate model, SLSNs could be powered by the spin-down of a nascent magnetar inside the supernova ejecta (Kasen \& Bildsten 2010), which may also produce X-ray emission (Metzger et al. 2014).

However, such emission is likely to be very sensitive to the exact properties of the CSM including density profile and homogeneity. In dense CSM environments, free-free absorption can suppress the radio emission at early times. Thus, chances for a detection are maximized by observing after maximum light
(Ofek et al. 2013). Hence, we observed iPTF15cyk thrice between 1 month and 4 months after discovery.

We also observed the location of iPTF15cyk with the Swift satellite (Gehrels et al. 2004) beginning at 18:12 UT on 2015 September 18 ( $\Delta t=4.3$ days after the GW trigger). We do not detect any emission with the on board X-Ray Telescope (XRT; Burrows et al. 2005) to a $3 \sigma$ limit of $<3.2 \times 10^{-3} \mathrm{ct} \mathrm{s}^{-1}$. Assuming a power-law spectrum with a photon index of $\Gamma=2$, this corresponds to an upper limit on the unabsorbed flux $(0.3-10.0 \mathrm{keV})$ of $f_{X}<1.3 \times 10^{-13} \mathrm{erg} \mathrm{cm}^{-2} \mathrm{~s}^{-1}$.

Simultaneously, we obtained images of the field with the Ultra-Violet Optical Telescope (UVOT; Roming et al. 2005) on board Swift in the $V, B, U, U V W 1, U V M 2$, and $U V W 2$ filters. No emission is detected at the location of iPTF15cyk. For a 3 !" 0 aperture we place the following magnitude limits (AB system) at this time: $V>19.29 ; B>19.81 ; U>20.62$; $U V W 1>21.61 ; U V M 2>22.27$; and $U V W 2>22.42$. These limits were derived using the revised UV zero points and timedependent sensitivity from Breeveld et al. (2011).

Additional spectroscopic follow-up of iPTF15cyk showed that it was a hydrogen-poor SLSN I (Quimby et al. 2011) at $z=$ 0.539 (Figure 4), similar to LSQ12dlf at $+16 \mathrm{~d}$ (Nicholl et al. 2014). The radio and X-ray upper limits were consistent with this classification. Given the high redshift, we concluded that this event was unrelated to GW150914. We note that the odds of finding a superluminous supernova were lower than the odds of finding other core-collapse or thermonuclear supernovae. The snapshot rate is only $\sim 0.2$ using the volumetric rate in Quimby et al. (2013; and assuming that SLSNs are brighter than -21 mag for 1 month). Moreover, we have a total of only six events with $z>0.5$ (out of 2650 spectroscopically classified supernovae) in the six years of operating PTF/iPTF. 


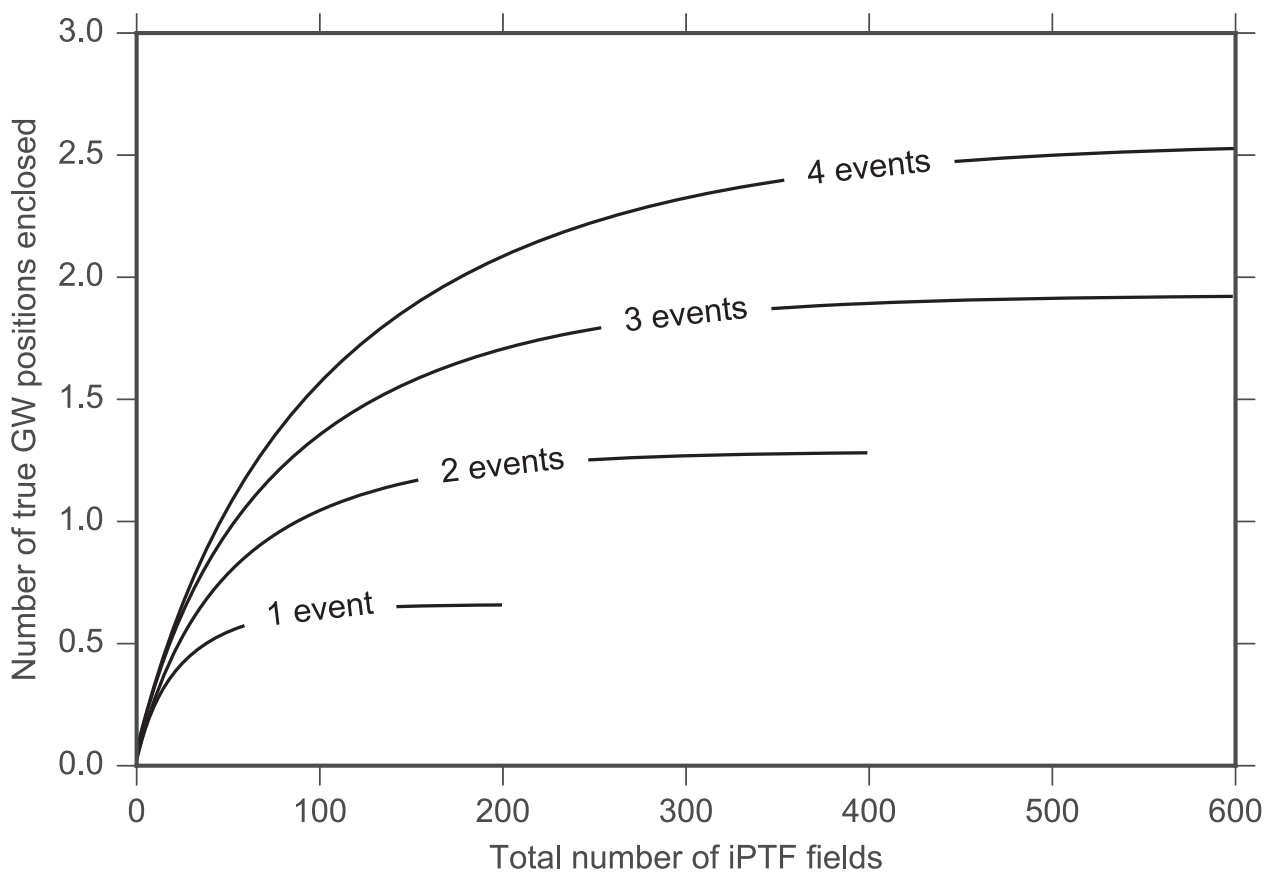

Figure 5. Simulation to compute the average number of times the true GW position would be enclosed in the iPTF imaged area as a function of the total number of iPTF fields imaged. Each iPTF field is $7.1 \mathrm{deg}^{2}$ and two $60 \mathrm{~s}$ images of 150 fields can be obtained in a night. Thus, we need to follow up two GW events to have at least one in our imaged area.

\section{A WAY FORWARD}

The post-detection era promises to be one of routine $\mathrm{GW}$ detections of binary neutron star mergers. With routine detections, the joint probability of $\sim \frac{1}{3}$ that the Sun $\left(\sim \frac{2}{3}\right)$, clouds $\left(\sim \frac{2}{3}\right)$, and latitude $\left(\sim \frac{3}{4}\right)$ simultaneously cooperate to identify the optical counterpart is not discouraging. Furthermore, given the location of Palomar Observatory in Southern California, relative to the location of the advanced LIGO interferometers, the time lag to respond is inherently less than an hour as we do not need to wait for the Earth to rotate (Kasliwal \& Nissanke 2014). Most of the GW150914 localization was not accessible from the northern night sky. However, based on our simulations (Singer et al. 2014), iPTF would include the true position of the GW source for an average of $\approx 1$ out of 2 events assuming a total of $100 \mathrm{iPTF}$ observations (see Figure 5; each observation is two $60 \mathrm{~s}$ exposures of $7.1 \mathrm{deg}^{2}$ ).

As advanced LIGO ramps up in GW sensitivity, we are undertaking both hardware and software upgrades to improve EM sensitivity. In 2017, we plan to commission the Zwicky Transient Facility (ZTF ${ }^{22}$; Kulkarni 2012; Bellm 2014), a $47 \mathrm{deg}^{2}$ camera on the Palomar 48 inch, with a 12 times higher volumetric survey speed than iPTF. This increase in survey speed enables a faster cadence and deeper search for the optical counterpart (e.g., $22 \mathrm{mag}$ in 10 minutes). The larger field of view may also be more robust to a shifting localization (e.g., for GW150914, our enclosed probability went from $2.5 \%$ in the initial map to $0.2 \%$ in the final map; see Abbott et al. 2016a). We are continuing to improve our software algorithms, e.g., better candidate filtering, image co-addition, and more optimal image subtraction (Zackay et al. 2016). We are continuing to complete our census of the local universe (CLU; D. Cook et al. 2016, in preparation) as this $200 \mathrm{Mpc}$ galaxy catalog serves as

\footnotetext{
${ }^{22}$ http://ptf.caltech.edu/ztf
}

the most severe filter for false positives (see examples in Nissanke et al. 2013). We have recently made some hardware changes to enable observing with the I-band filter, and possibly provide some constraints on kilonova models predicting red emission (Metzger \& Fernández 2014; Kasen et al. 2015).

Among the various models for EM emission from binary neutron star mergers, free neutron decay gives the most luminous optical counterpart (Figure 3; Metzger et al. 2015). Varying free neutron mass and opacity suggests that this counterpart may fade quickly, as much as $4 \mathrm{mag}$ in $24 \mathrm{hr}$. Thus, we are also systematizing our follow-up with the Global Relay of Observatories Watching Transients Happen $\left(\mathrm{GROWTH}^{23}\right)$ program. The combination of a longitudinally distributed network of telescopes as well as multi-wavelength follow-up (VLA and Swift) should effectively filter candidates on a $24 \mathrm{hr}$ timescale. Obtaining a timely light curve, spectra, and spectral energy distribution will unravel both the astrophysics and the astrochemistry of the EM counterpart. With this first gravitational-wave detection, the 21st century gold rush (Kasliwal 2013) has officially begun!

Based on observations obtained with the Samuel Oschin Telescope 48 inch and the 60 inch Telescope at the Palomar Observatory as part of the iPTF project, a scientific collaboration among the California Institute of Technology, Los Alamos National Laboratory, the University of Wisconsin-Milwaukee, the Oskar Klein Center, the Weizmann Institute of Science, the TANGO Program of the University System of Taiwan, and the Kavli Institute for the Physics and Mathematics of the universe. M.M.K., R.L., and Y.C. acknowledge support from the National Science Foundation PIRE program grant 1545949. A.A.M. acknowledges support from the Hubble Fellowship HST-HF-51325.01. P.E.N. and Y.C. acknowledge support from the DOE under grant DE-AC02-05CH11231, Analytical Modeling for Extreme-Scale Computing Environments. The

\footnotetext{
${ }^{23}$ http://growth.caltech.edu
} 
Table 3

Observations Log

\begin{tabular}{|c|c|c|c|c|c|c|c|}
\hline Index & PTF Field ID & Central RA (J2000) & Central DEC (J2000) & Observation Time (UTC) & Filter & Airmass & Limiting mag $(5 \sigma)$ \\
\hline 1 & 3050 & 132.000 & 7.875 & 2015 Sep 17 12:13:20 & $\mathrm{R}$ & 2.7 & 20.4 \\
\hline 1 & 3050 & 132.000 & 7.875 & 2015 Sep 24 12:02:27 & $\mathrm{R}$ & 2.3 & 20.3 \\
\hline 1 & 3050 & 132.000 & 7.875 & 2015 Sep 24 12:13:30 & $\mathrm{R}$ & 2.2 & 20.3 \\
\hline 2 & 3154 & 129.808 & 10.125 & 2015 Sep 17 12:11:38 & $\mathrm{R}$ & 2.4 & 20.4 \\
\hline 3 & 3155 & 133.269 & 10.125 & 2015 Sep 17 12:15:01 & $\mathrm{R}$ & 2.7 & 20.2 \\
\hline 4 & 3257 & 127.573 & 12.375 & 2015 Sep 17 12:03:09 & $\mathrm{R}$ & 2.3 & 20.6 \\
\hline 4 & 3257 & 127.573 & 12.375 & 2015 Sep 17 12:33:42 & $\mathrm{R}$ & 1.9 & 19.6 \\
\hline 5 & 3258 & 131.068 & 12.375 & 2015 Sep 17 12:09:56 & $\mathrm{R}$ & 2.5 & 20.4 \\
\hline 5 & 3258 & 131.068 & 12.375 & 2015 Sep 17 12:40:29 & $\mathrm{R}$ & 2.0 & 18.2 \\
\hline 6 & 3259 & 134.563 & 12.375 & 2015 Sep 17 12:16:43 & $\mathrm{R}$ & 2.6 & 20.1 \\
\hline 7 & 3359 & 125.294 & 14.625 & 2015 Sep 17 12:01:27 & $\mathrm{R}$ & 2.1 & 20.6 \\
\hline 7 & 3359 & 125.294 & 14.625 & 2015 Sep 17 12:32:00 & $\mathrm{R}$ & 1.8 & 20.0 \\
\hline 8 & 3360 & 128.824 & 14.625 & 2015 Sep 17 12:04:51 & $\mathrm{R}$ & 2.3 & 20.5 \\
\hline 8 & 3360 & 128.824 & 14.625 & 2015 Sep 17 12:35:24 & $\mathrm{R}$ & 1.9 & 19.3 \\
\hline 9 & 3361 & 132.353 & 14.625 & 2015 Sep 17 12:08:15 & $\mathrm{R}$ & 2.5 & 20.4 \\
\hline 9 & 3361 & 132.353 & 14.625 & 2015 Sep 17 12:38:47 & $\mathrm{R}$ & 2.0 & 18.6 \\
\hline 10 & 3362 & 135.882 & 14.625 & 2015 Sep 17 12:18:25 & $\mathrm{R}$ & 2.6 & 20.2 \\
\hline 11 & 3459 & 119.406 & 16.875 & 2015 Sep 17 11:52:56 & $\mathrm{R}$ & 1.9 & 20.8 \\
\hline 11 & 3459 & 119.406 & 16.875 & 2015 Sep 17 12:23:31 & $\mathrm{R}$ & 1.6 & 20.7 \\
\hline 12 & 3460 & 122.970 & 16.875 & 2015 Sep 17 11:56:21 & $\mathrm{R}$ & 2.0 & 20.6 \\
\hline 12 & 3460 & 122.970 & 16.875 & 2015 Sep 17 12:26:55 & $\mathrm{R}$ & 1.7 & 20.4 \\
\hline 13 & 3461 & 126.535 & 16.875 & 2015 Sep 17 11:59:46 & $\mathrm{R}$ & 2.1 & 20.6 \\
\hline 13 & 3461 & 126.535 & 16.875 & 2015 Sep 17 12:30:19 & $\mathrm{R}$ & 1.8 & 20.1 \\
\hline 13 & 3461 & 126.535 & 16.875 & 2015 Sep 18 11:39:19 & $\mathrm{R}$ & 2.4 & 20.6 \\
\hline 13 & 3461 & 126.535 & 16.875 & 2015 Sep 18 12:00:29 & $\mathrm{R}$ & 2.1 & 20.7 \\
\hline 14 & 3462 & 130.099 & 16.875 & 2015 Sep 17 12:06:33 & $\mathrm{R}$ & 2.2 & 20.6 \\
\hline 14 & 3462 & 130.099 & 16.875 & 2015 Sep 17 12:37:05 & $\mathrm{R}$ & 1.8 & 19.0 \\
\hline 15 & 3560 & 120.600 & 19.125 & 2015 Sep 17 11:54:38 & $\mathrm{R}$ & 1.8 & 20.8 \\
\hline 15 & 3560 & 120.600 & 19.125 & 2015 Sep 17 12:25:13 & $\mathrm{R}$ & 1.6 & 20.6 \\
\hline 15 & 3560 & 120.600 & 19.125 & 2015 Sep 18 11:13:55 & $\mathrm{R}$ & 2.3 & 20.6 \\
\hline 15 & 3560 & 120.600 & 19.125 & 2015 Sep 18 11:37:37 & $\mathrm{R}$ & 2.0 & 20.7 \\
\hline 16 & 3561 & 124.200 & 19.125 & 2015 Sep 17 11:58:04 & $\mathrm{R}$ & 2.0 & 20.7 \\
\hline 16 & 3561 & 124.200 & 19.125 & 2015 Sep 17 12:28:36 & $\mathrm{R}$ & 1.7 & 20.4 \\
\hline 17 & 3658 & 115.714 & 21.375 & 2015 Sep 17 11:49:31 & $\mathrm{R}$ & 1.7 & 20.9 \\
\hline 17 & 3658 & 115.714 & 21.375 & 2015 Sep 17 12:20:08 & $\mathrm{R}$ & 1.5 & 20.9 \\
\hline 17 & 3658 & 115.714 & 21.375 & 2015 Sep 18 10:50:49 & $\mathrm{R}$ & 2.3 & 20.6 \\
\hline 17 & 3658 & 115.714 & 21.375 & 2015 Sep 18 11:12:13 & $\mathrm{R}$ & 2.0 & 20.7 \\
\hline 17 & 3658 & 115.714 & 21.375 & 2015 Sep 19 11:44:39 & $\mathrm{R}$ & 1.6 & 20.7 \\
\hline 17 & 3658 & 115.714 & 21.375 & 2015 Sep 19 12:26:26 & $\mathrm{R}$ & 1.4 & 20.8 \\
\hline 18 & 3659 & 119.388 & 21.375 & 2015 Sep 17 11:51:13 & $\mathrm{R}$ & 1.8 & 20.9 \\
\hline 18 & 3659 & 119.388 & 21.375 & 2015 Sep 17 12:21:50 & $\mathrm{R}$ & 1.5 & 20.8 \\
\hline
\end{tabular}

National Radio Astronomy Observatory is a facility of the National Science Foundation operated under cooperative agreement by Associated Universities, Inc. A.C. and N.P. acknowledge support from NSF CAREER award 1455090. Part of the research was carried out at the Jet Propulsion Laboratory, California Institute of Technology, under a contract with NASA. M.M.K. thanks Brian Metzger for providing us theoretical light curves for neutron-powered precursors. We thank the referee for constructive feedback.

\section{APPENDIX \\ SIMULATION}

We simulated optimal P48 scheduling for GW events in order to quantify the tradeoff between observing time and contained probability during $\mathrm{O} 1$. We took all $N_{i}=250$ simulated BNS merger sky maps, enumerated as $i=1, \ldots, N_{i}$, from the " 2015 " scenario in Singer et al. (2014). We shifted the GMSTs of the events by random integer multiples of 1 day so that the sky maps remained unchanged in equatorial coordinates but the event times were uniformly distributed throughout the planned time span of O1, 2015 October 1 through 2016 January 1.

We divided each day into $N_{j}=864$ time blocks labeled $j=1, \ldots, N_{j}$, each $100 \mathrm{~s}$ long. We divided the sky into 12,288 equal-area HEALPix pixels (Górski et al. 2005), each spanning an area of $3.4 \mathrm{deg}^{2}$. (This is about half of the FOV of iPTF; the next coarser resolution would be about twice the FOV of iPTF because HEALPix pixel densities are related by powers of 4.)

Assuming ideal weather conditions, we calculated an observability mask as a function of time and sky position in the form of a $N_{j} \times N_{k}$ element bitmap for each one-day period following a simulated GW event. A time and position was considered observable under the following criteria:

1. The altitude of the Sun is $<-12^{\circ}$.

2. The hour angle is $<6.5^{\mathrm{h}}$. 
3. The airmass is $<2.5$ (e.g., the altitude is $>23.5$ ).

4. The separation from the center of the Moon is $>10^{\circ} \times\left(\text { Moon phase } / 180^{\circ}\right)^{2}$.

For each event $i$, we planned an optimal one-night observing schedule by casting it as a canonical problem in graph theory: finding the maximum matching of an edge-weighted bipartite graph. The left nodes of the graph were the $N_{j}$ time blocks, and the right nodes were the $N_{k}$ pixels. The edge $j k$ was present if pixel $k$ was observable at time $j$. The weight of edge $j k$ was the probability from the sky map that the GW source is contained in pixel $k,{ }_{P i}(k)$. We found the global optimum observing plan using the LEMON library ${ }^{24}$. We ranked the resulting schedules from most to least probable, yielding the sequence $\left\{P_{i}^{\prime}(k)\right\}_{k}$.

In Figure 5, we plot the expected number of GW sources that would be successfully imaged as a function of the number of iPTF fields, if we followed up $N=1,2,3$, or $4 \mathrm{GW}$ candidates selected randomly out of the Singer et al. (2014) sample. (The number of fields plotted on the horizontal axis is actually half of the number of scheduled tiles in order to correct for the fact that the HEALPix tiles are half of the size of the iPTF FOV.)

Unsurprisingly, we find that we need to follow up at least two events to have a good chance of containing the position of one source: the bimodality of two-detector O1 sky maps means that only half of the GW localization region is observable for any one event. The improvement in contained probability reaches a point of diminishing returns at 100-150 iPTF fields per GW event. The growth of the containment probability with each added field generally flattens out by 150 fields. Assuming two $100 \mathrm{~s}$ exposures per field, this implies a maximum of $5.5-8.3 \mathrm{hr}$, or an entire night of iPTF P48 observations, per GW event.

In the specific case of GW150914, the Sun-angle and declination constraints severely constrained the visibility window. Our detailed observing log is presented in Table 3.

\section{REFERENCES}

Aasi, J., Abadie, J., Abbott, B. P., et al. 2014, ApJS, 211, 7

Abbott, B., Abbott, R., Abbott, T. D., et al. 2016a, https://dcc.ligo.org/LIGOP1500227/public/main

Abbott, B., Abbott, R., Abbott, T. D., et al. 2016b, PhRvL, 116, 061102

Abbott, B. P., Abbott, R., Abbott, T. D., et al. 2013, LRR, 19, 1

Bellm, E. 2014, in The Third Hot-wiring the Transient universe Workshop, ed. P. R. Wozniak et al., 27
Berry, C. P. L., Mandel, I., Middleton, H., et al. 2015, ApJ, 804, 114 Breeveld, A. A., Landsman, W., Holland, S. T., et al. 2011, AIP Conf. Ser. 1358 , GAMMA RAY BURSTS 2010, ed. J. E. McEnery, J. L. Racusin, \& N. Gehrels (Melville, NY: AIP), 373

Brink, H., Richards, J. W., Poznanski, D., et al. 2013, MNRAS, 435, 1047

Burrows, D. N., Hill, J. E., Nousek, J. A., et al. 2005, SSRv, 120, 165

Connaughton, V., Burns, E., Goldstein, A., et al. 2016, ApJL, in press (arXiv:1602.03920)

Evans, P. A., Kennea, J. A., Barthelmy, S. D., et al. 2016, MNRAS, 460, L40

Faber, S. M., Phillips, A. C., Kibrick, R. I., et al. 2003, Proc. SPIE, 4841,1657

Gehrels, N., Chincarini, G., Giommi, P., et al. 2004, ApJ, 611, 1005

Górski, K. M., Hivon, E., Banday, A. J., et al. 2005, ApJ, 622, 759

Howell, D. A., Sullivan, N., Perrett, K., et al. 2005, ApJ, 634, 1190

Kasen, D., \& Bildsten, L. 2010, ApJ, 717, 245

Kasen, D., Fernández, R., \& Metzger, B. D. 2015, MNRAS, 450, 1777

Kasliwal, M. M. 2013, Sci, 340, 555

Kasliwal, M. M., Cenko, S. B., Cao, Y., et al. 2015, GCN, 18341, 1

Kasliwal, M. M., \& Nissanke, S. 2014, ApJL, 789, L5

Kulkarni, S. R. 2012, arXiv:1202.2381

Law, N. M., Kulkarni, S. R., Dekany, R. G., et al. 2009, PASP, 121, 1395

Li, W., Leaman, J., Chornock, R., et al. 2011, MNRAS, 412, 1441

LIGO Scientific Collaboration, \& Virgo 2015, GCN, 18330, 1

LIGO Scientific Collaboration, \& Virgo 2016, GCN, 18858, 1

Metzger, B. D., Bauswein, A., Goriely, S., \& Kasen, D. 2015, MNRAS, 446, 1115

Metzger, B. D., \& Fernández, R. 2014, MNRAS, 441, 3444

Metzger, B. D., Vurm, I., Hascoët, R., \& Beloborodov, A. M. 2014, MNRAS, 437, 703

Nicholl, M., Smartt, S. J., Jerkstrand, A., et al. 2014, MNRAS, 444, 2096

Nissanke, S., Kasliwal, M., \& Georgieva, A. 2013, ApJ, 767, 124

Nugent, P., Cao, Y., \& Kasliwal, M. M. 2015, Proc. SPIE, 9397

Ofek, E. O., Fox, D., Cenko, S. B., et al. 2013, ApJ, 763, 42

Palliyaguru, N., \& Corsi, A. 2016, GCN, 18914, 1

Perley, R., Napier, P., Jackson, J., et al. 2009, IEEEP, 97, 1448

Quimby, R. M., Kulkarni, S. R., Kasliwal, M. M., et al. 2011, Natur, 474, 487

Quimby, R. M., Yuan, F., Akerlof, C., \& Wheeler, J. C. 2013, MNRAS, 431, 912

Rana, J., Singhal, A., Gadre, B., Bhalerao, V., \& Bose, S. 2016, ApJ, submitted (arXiv:1603.01689)

Rebbapragada, U. 2014, in in The Third Hot-wiring the Transient Universe Workshop, ed. P. R. Wozniak et al., 205

Roming, P. W. A., Kennedy, T. E., Mason, K. O., et al. 2005, SSRv, 120, 95

Singer, L. P., Cenko, S. B., Kasliwal, M. N., et al. 2013, ApJL, 776, L34

Singer, L. P., Kasliwal, M. M., Cenko, S. B., et al. 2015, ApJ, 806, 52

Singer, L. P., Kasliwal, M. M., Cenko, S. B., et al. 2015, GCN, 18337, 1

Singer, L. P., Price, L. R., Farr, B., et al. 2014, ApJ, 795, 105

Smartt, S. J., Chambers, K. C., Smith, K. W., et al. 2016, MNRAS, submitted (arXiv:1602.04156)

Soares-Santos, M., Kessler, R., Berger, E., et al. 2016, ApJL, 823, L33

Zackay, B., Ofek, E. O., \& Gal-Yam, A. 2016, arXiv:1601.02655

\footnotetext{
${ }^{24}$ http://lemon.cs.elte.hu/pub/doc/1.2.3/a00219.html
} 